\title{
Phase decomposition and reversion in Al-Li alloys
}

\author{
K. OSAMURA and H. OKUDA
}

Department of Metallurgy, Kyoto University, Sakyo-ku, Kyoto 606, Japan

\begin{abstract}
After overview on the behaviour of first order order/disorder phase transformation based on the instability diagrams, the results of in-situ SR-SAS measurements have been reported in order to clarify the process of transformation, the growth mechanism of metastable $L 1_{2}$ phase and its coarsening as well as the reversion process in Al-Li alloys. The results were compared with computer simulation by using kinetic Ising model with interaction up to the second nearest neighbour. The relationship between local ordering and clustering and the scaling behaviour of structure factor have been discussed in detail, especially at very early stage of phase decomposition and during rapid reversion at high temperatures.
\end{abstract}

\section{Introduction}

Kinetics of phase decomposition with ordering is a recent topics in the research field of diffusional phase transformation. As a typical example, the phase decomposition behaviour in Al-Li metallic system has been paid much attention, where the first order order/disorder phase transformation takes place. As discussed by various authors ${ }^{1)-4)}$, several decomposition paths are able to be proposed depending on composition and temperature. The disordered FCC phase transforms to the ordered $\mathrm{L} 1_{2}$ type $\delta$ ' phase of which unit cell comprises four lattice points of one Li site on $(0,0,0)$ and three Al sites on $(0,1 / 2,1 / 2),(1 / 2,0,1 / 2)$ and $(1 / 2,1 / 2,0)$. Using long range order parameter, $\eta$, the solute concentration is defined on two types of lattice site: $c(1-\eta)$ on $\mathrm{Al}$ site and $\mathrm{c}(1+3 \eta)$ on $\mathrm{Li}$ site. Here Li atoms occupy one simple cubic lattice site represented by $(0,0,0)$. There exist 4 similar sublattices, when we take different origin.

According to Khachaturyan ${ }^{1)}$, the internal energy of the system is expressed as a function of compositions and long range order parameter as given by the equation,

$$
E(c, \eta)=\frac{N}{2}\left\{2 f^{\circ} c+\left[V(0)+3 V(100) \eta^{2}\right] c^{2}\right\},
$$

where $f^{o}$ is the free energy in the dilute solution limit, and $V(0)$ and $V(100)$ are the first and second nearest neighbour interation parameters. The tendency of ordering or phase decomposition is predicted using these two interaction parameters. In the case of negative $V(0)$, phase decompostion takes place, where compositional modulation appears in space. The region with negative $V(100)$ corresponds to ordering process. In the sector of $V(0)<0$ and $V(100)<0$, ordeirng and phase decomposition overlap. The present Al-Li system locates in the region of $V(100)<0$, but has an alternative sign of $\mathrm{V}(0)$ depending on the theory proposed. 
Figure 1 is a phase diagram for the present Al-Li alloy system calculated by Garland and Sanchez ${ }^{5)}$, where the sign is negative for both parameters, $\mathrm{V}(0)$ and $\mathrm{V}(100)$. Here three instability temperatures have the follwoing meaning; $\mathrm{T}_{i}^{-}$is instability of $\mathrm{FCC}$ with respect to atomic ordering to $\mathrm{L} 1_{2}$; $\mathrm{T}_{c s}$ is instability of $\mathrm{L} 1_{2}$ with respect to phase separation; $\mathrm{T}_{o}$ is temperature at which the free energy of $\mathrm{FCC}$ is equal to that of $\mathrm{L} 1_{2}$. When the alloy is quenched to two phase field, the decomposition path is deduced from the free energy composition diagram. Usually the disordered state is realized by rapid quenching.

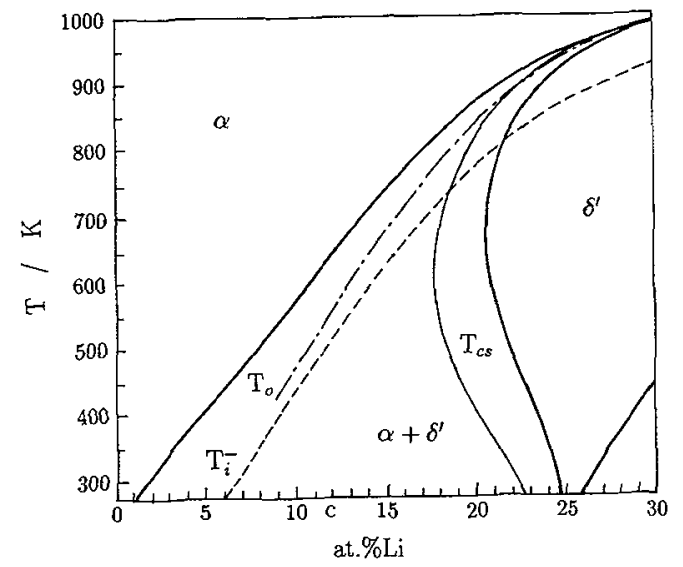

Fig.1 Metastable phase diagram of Al-Li binary system calculated by Garland and Sanchez).

After that, as discussed by Soffa and Laughlin ${ }^{2)}$, the transformation path changes as a function of composition and order parameter. On ordering process from the disordered state, one case corresponds to the contiunous ordering, and another one is the congruent ordering accomanying with the nucleation and growth of oredered phase. As a summary, five possible paths for ordering and decomposition kinetics are proposed as listed below.
1. $\alpha\left(\mathrm{c}_{o}\right)=$ N.G. $\Rightarrow \delta^{\prime}\left(\mathrm{c}_{\delta}\right)+\alpha\left(\mathrm{c}_{\alpha}\right)$
2. $\alpha\left(\mathrm{c}_{o}\right)=$ cong. O. $\Longrightarrow \delta^{\prime}\left(\mathrm{c}_{o}\right)=$ N.G. $\Rightarrow \delta^{\prime}\left(\mathrm{c}_{\delta}\right)+\alpha\left(\mathrm{c}_{\alpha}\right)$
3. $\alpha\left(\mathrm{c}_{o}\right)=$ cont. O. $\Rightarrow \delta^{\prime}\left(\mathrm{c}_{o}\right)=$ N.G. $\Longrightarrow \delta^{\prime}\left(\mathrm{c}_{\delta}\right)+\alpha\left(\mathrm{c}_{\alpha}\right)$
4. $\alpha\left(\mathrm{c}_{o}\right)=$ cong. O. $\Longrightarrow \delta^{\prime}\left(\mathrm{c}_{o}\right)=$ S.D. $\Longrightarrow \delta^{\prime}\left(\mathrm{c}_{1}\right)+\delta^{\prime}\left(\mathrm{c}_{2}\right)=$ D.O. $\Longrightarrow \delta^{\prime}\left(\mathrm{c}_{\delta}\right)+\alpha\left(\mathrm{c}_{\alpha}\right)$
5. $\alpha\left(\mathrm{c}_{o}\right)=$ cont. O. $\Longrightarrow \delta^{\prime}\left(\mathrm{c}_{o}\right)=$ S.D. $\Longrightarrow \delta^{\prime}\left(c_{1}\right)+\delta^{\prime}\left(c_{2}\right)=$ D.O. $\Longrightarrow \delta^{\prime}\left(\mathrm{c}_{\delta}\right)+\alpha\left(\mathrm{c}_{\alpha}\right)$

The first path is that the disordered phase $\alpha\left(c_{o}\right)$ decomposes directly into two phases, $\delta^{\prime}\left(c_{\delta}\right)+\alpha\left(c_{\alpha}\right)$ by nucleation and growth ( N.G. ) mechanism. In the 4th path, firstly congruent ordering takes place, and the ordered phase decomposes by spinodal decompostion ( S.D. ) into two ordered phases. And then one phase transforms into the disordered phase by disordering ( D.O. ) process.

In the present study, the in-situ small angle scattering measurements as wll as the diffuse scattering measurements have been performed in order to clarify the process of ordering and decomposition, and the growth mechanism of metastable $\mathrm{L}_{2}$ phase as well as the reversion process in the Al-Li alloy system. The results were compared with the result of computer simulation by using kinetic Ising model.

\section{Computer simulation based on Ising model}

Computer simulation has been carried out by using FACOM M1800 system at Kyoto University in order to investigate the kinetics of phase decompositions in the Ising lattice system. The size of the system used here was $40 \times 40 \times 40 \mathrm{FCC}$ unit cells with solute concentration of $c=0.12$. Two 
interaction parameters were selected as $\mathrm{J}_{1}=-1.0$, and $\mathrm{J}_{2}=0.5$, which are the same with the phase diagram calculation by Garland and Sanchez ${ }^{5}$. The details will be published elsewhere ${ }^{6)}$.

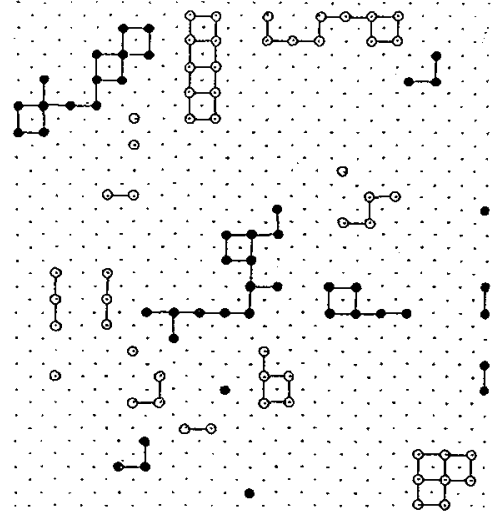

Fig.2 Snapshot of solute atom distribution on (100) plane of FCC lattice during decompostion at $T=1.0$ after 15.4 MCS.

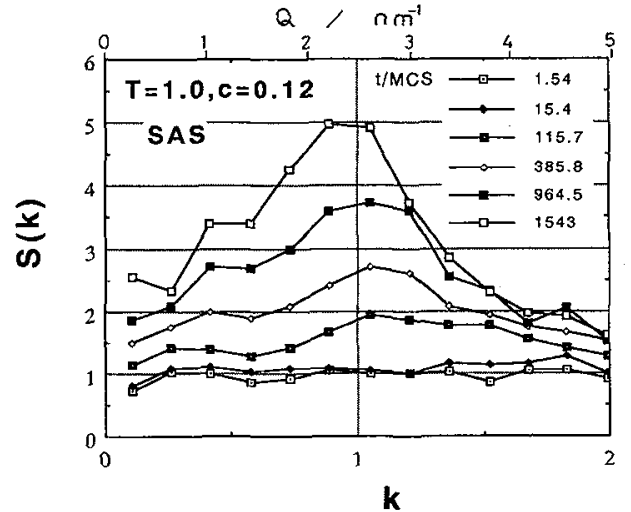

Fig.3 Calculated small angle scattering intensity as a function of scattering vector in the Ising system. $Q$ is the scattering vector relevant to the Al-Li system.

Figure 2 shows a snapshot on (100) plane of FCC lattice during decomposition at $\mathrm{T}=1.0$ after 15.4 MCS. Two kinds of sign indicate solute atoms on the different sublattice. The coordination number is defined as the number of solute atoms occupying the nearest neighbour site around individual solute atom. The number 2 indicates one-dimensional solute atoms chain, the number 3 is the ladder, the number 4 is two dimensional network and the higher number corresponds to three dimensional cluster. Up to $30 \mathrm{MCS}$, the average number remains less than 3 , which means that the structure is mostly one or two-dimensional. When the annealing time increases exceeding $10^{3} \mathrm{MCS}$, the number becomes larger than 4 indicating an appearance of three dimensional clusters.

Figure 3 shows the scattering vector dependence of structure factor at small angle scattering region. At a short annealing time up to $15 \mathrm{MCS}$, no scattering intensity is observed. When the time exceeds $10^{2} \mathrm{MCS}$, the intensity increased remarkably. The position of intensity maximum, $\mathrm{k}_{m}$ shifts towards the lower scattering vector region with time. The $k_{m}$ approaches about $2 \mathrm{~nm}^{-1}$ at 1543 MCS.

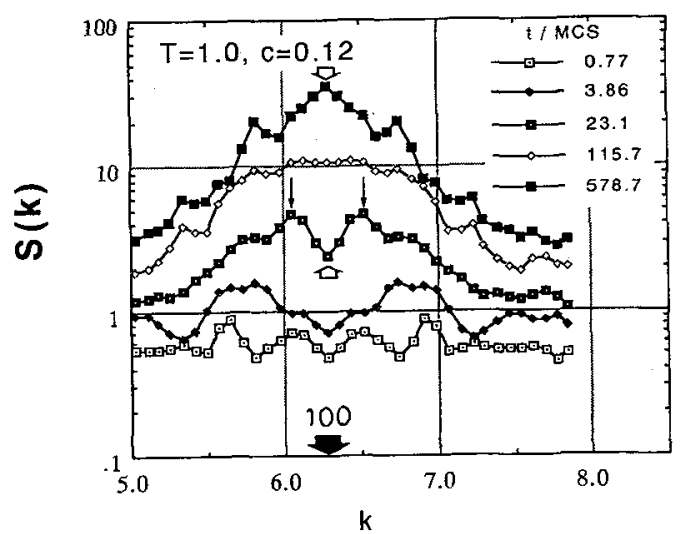

Fig.4 Calculated diffuse scattering intensity around 100 ordered spot as a function of scattering vector in the Ising system.

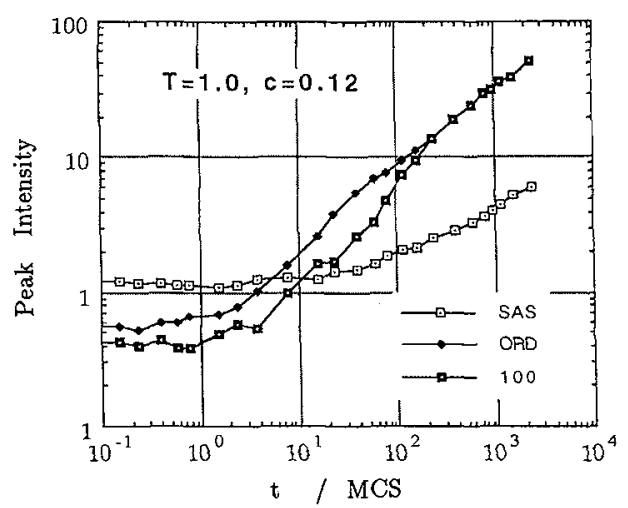

Fig.5 Change of calculated peak intensity around 000 and 100 reciprocal lattice points as a function of time in the Ising system. 
The structure factor around 100 ordered spot is shown in Fig. 4. Even at short time less than 15 MCS, the fine structure is visible. Looking at the scattering intensity at 23.1 MCS, a minimum appears at the exact 100 position surrounding by the maxima. When the time exceeds $100 \mathrm{MCS}$, the scattering intensity becomes maximum at the exact 100 position.

Figure 5 shows the changes of peak intensity as a function of annealing time. SAS intensity is very weak and does not change up to $30 \mathrm{MCS}$. but the intensity of 100 ordered spot starts to increase at time of 3 MCS. The structure change can be, therefore divided into 3 stages in the following: at stage I, one dim. short range ordering ( SRO ) developes without compositional fluctuation, stage II corresponds to development of two-dim SRO, stage III is growth of three-dim. L $1_{2}$ ordered phase.

When the spherical clusters of ordered phase disperse homogeneously, the structure factor is given at around the reciprocal lattice point of $h 00=000$ or h00 $=100$ by the following similar equation,

$$
\begin{gathered}
S_{h 00}(\mathbf{k})=\left|A_{h 00}(\mathbf{k})\right|^{2}\left[1-v \rho_{o} \Phi(\mathbf{k l}) P_{h 00}\right] \\
P_{h 00}=p_{0}+p_{2}+(-1)^{h}\left[p_{1}+p_{3}\right] \exp (i \pi \Delta \mathbf{k})
\end{gathered}
$$

where $\mathrm{A}(\mathrm{k})$ is the average structure factor for individual clusters and $\Phi(\mathbf{k l})$ is the interparticle interference factor, $\mathrm{v}$ and $\rho$ are the average volume and the number density of clusters, and $\Delta \mathrm{k}=$ $\mathbf{k}-\mathbf{k}_{h 00}$. The $\mathrm{p}_{i}$ is the probability of finding a cluster on respective sublattice, where there are four choices selecting the vector reaching the gravity center of each cluster. The numbers $0,1,2,3$ indicate different sublattice. When they distribute randomly, $P_{100}$ becomes zero. This means the interparticle interference effect disappear in the diffuse scattering intensity around the 100 ordered spot. When there exists a deviation of cluster distribution with respect to specific sublattice, it is expected that the minimum appears at the exact position of ordered 100 spot. On the other hand, the intensity maximum appears in the small angle scattering regardless a change of sublattice distribution.

\section{In-situ SR-SAS measurements during phase decomposition}

The alloy used here was Al-11.8at\%Li alloy. It was solution treated at $623 \mathrm{~K}$ and quenched into ice water. In order to investigate the structure change during phase decomposition, in-situ SRSAS measurements have been performed at A15 facility of $\mathrm{KEK}$, Tsukuba during isothermal aging typically at 533 and $413 \mathrm{~K}$. The experimental details have been reported ${ }^{4)}$.

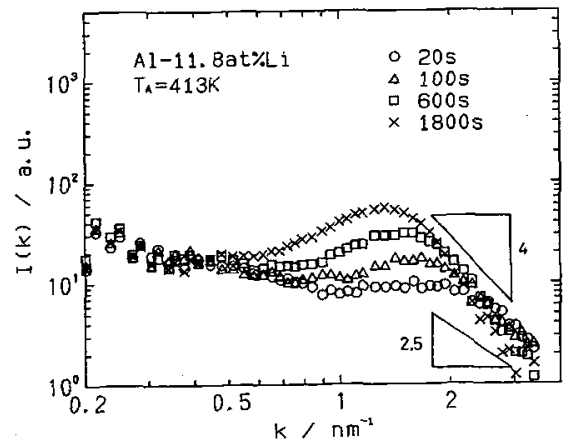

Fig.6 Small snagle scattering intensity as a function of scattering vector for $A l$ 11.8 at\% Li alloy during isothermal aging at $413 \mathrm{~K}$.

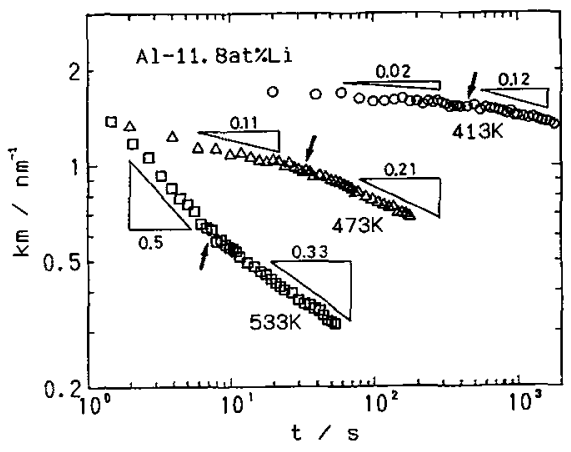

Fig.7 Change of $k_{m}$ as a function of annealing time for Al-11.8at\%Li alloy aged at various temperatures.

After the first $3 \mathrm{sec}$ aging at $533 \mathrm{~K}$, a clear SAS intensity was observed and it increased with time and the $k_{\max }$ shifted towards lower scattering vector region. The intensity is usually proportional to $\mathrm{k}^{-m}$ at the high scattering vector region. Here $\mathrm{m}$ was found to 4 . As a result, the characteristics of 
SAS intensity change at $533 \mathrm{~K}$ is able to be explained by the decomposition regime due to the N.G. of ordered $\delta$ ' phase.

As shown in Fig. 6, the scattering intensity for the alloy aged at $413 \mathrm{~K}$ is relatively weak. $\mathrm{m}$ was found to be 2.5 at the early time of $20 \mathrm{sec}$, but increased to 4 with increasing aging time. Figure 7 shows the change of $k_{m}$ as a function of aging time for the specimens aged at three different temperatures. The slope changes with time, when we draw a straight line, obviously two slopes are present for each data depending on time. For instance, at $413 \mathrm{~K}$, the initial slope is 0.02 , but the second slope becomes 0.12 . Such a change of the slope suggests the alternation of decomposition regime ${ }^{4)}$. $\mathrm{k}_{m}$ becomes about $2 \mathrm{~nm}^{-1}$ at very early aging time. This number corresponds to the late stage III of computer simulation as shown in Fig. 3. In order to investigate the early stage as made clear by the computer simulation, much intensive X-ray source and more sophisticated detector system are suggested to be constructed in near future.

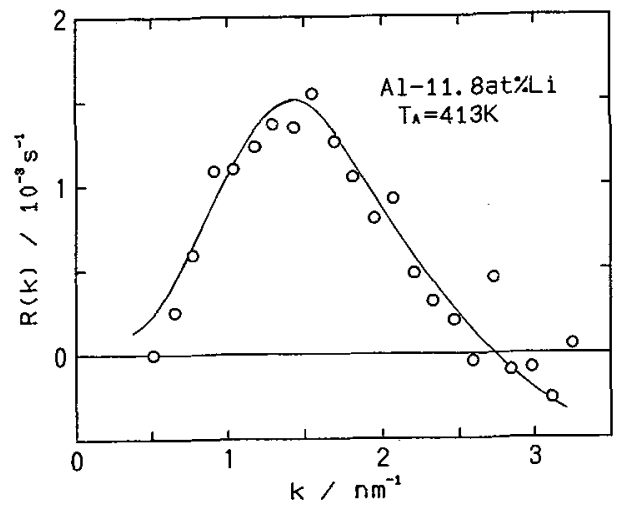

Fig.8 Change of amplification factor as a function of wavenumber for Al $11.8 a \mathrm{t} \% \mathrm{Li}$ alloy aged at $413 \mathrm{~K}$.

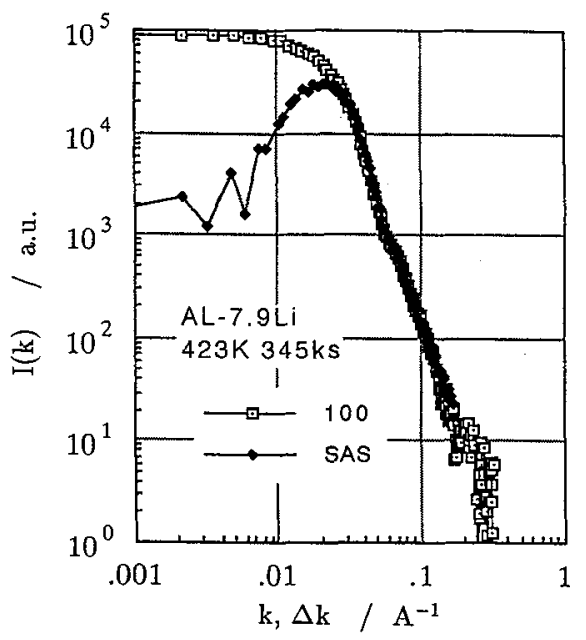

Fig.9 Comparison between $S A S$ and diffuse scattering intensities for Al -7.9 at\% Li alloy aged at $423 \mathrm{~K}$ for $345 \mathrm{ks}$.

The time dependence of the logarithmic intensity at a constant scattering vector was examined for the first stage of $413 \mathrm{~K}$ aging. The well-defined linear relationship with time was confirmed. According to a linear spinodal theory, the logarithmic intensity is given as a linear function of time, where the proportional constant is called the amplification factor $R(t)$. The amplification factor is plotted as a function of wave number as shown in Fig. 8. On increasing $\mathrm{k}$, the factor reaches a maximum and decreases and goes negative. The ratio of positions at the maximum to the zero $R(t)$ becomes 1.9 , which is slightly larger than the linear theory. From those reasons mentioned above, it was suggested that the spinodal decomposition takes place within the ordered state, when the alloy was aged at $413 \mathrm{~K}$. It was, however not possible to specify whether the congruent or the continuous ordering operates.

Figure 9 shows a comparison between SAS intensity around 100 ordered spot for Al -7.9 at\% $\mathrm{Li}$ alloy aged for long time. While the maximum appears in SAS intensity, the diffuse scattering intensity decreased monotonically. From this observation, the well-developed $\delta$ ' precipitates by long time aging are suggested to distribute randomly with respect to sublattice occupation.

\section{Reversion kinetics of $\delta^{\prime}$ phase}

When the alloy is held at high temperature above the solvus line just after the long-time low temperature aging at $423 \mathrm{~K}$ for $86 \mathrm{ks}$, $\delta^{\prime}$ precipitates shall resolve in the matrix. The structure 
change during this reversion process has been investigated by in-situ SAS measurements ${ }^{7)}$. It was very prominent that Guinier radius decreases linearly with time. Here it is supposed that the change of volume fraction is proportional to that of the average volume of precipitates. Then the follwoing function is derived,

$$
f(X)=1-\left(\frac{U X}{X+V}\right)^{1 / 3}=\frac{k}{R_{o}} t
$$

where $X$ is the relative integrated intensity, $Q / Q_{o}, R_{o}$ is the initial value of particle radius, $k, U$ and $\mathrm{V}$ are the constant. As shown in Fig. 10, the function was experimentally confirmed to increase linearly with time. From this fact, it was suggested that the dissolution reaction at the interface is the rate controlling process.

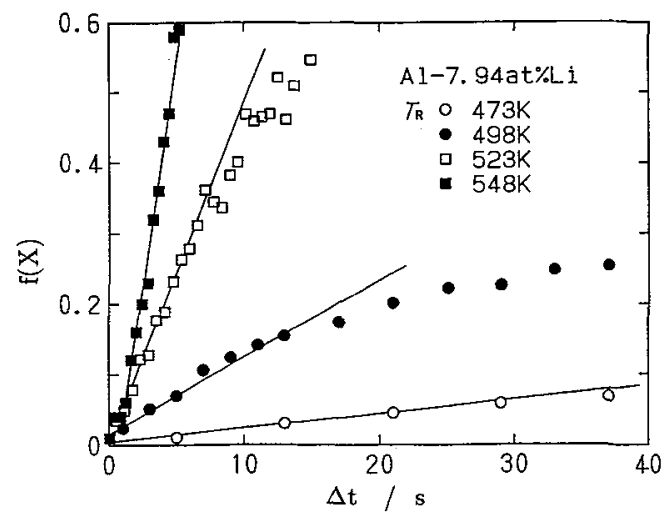

Fig.10 Change of $f(X)$ as a function of reversion time for Al-7.9at\% Li alloy reverted at various temepratures.

\section{Summary}

The present results are summarized as follows: (1) The structure change on the Ising lattice could be divided into three stages of one-dim. SRO, the development of two-dim. SRO and the growth of three-dim. $\mathrm{L1}_{2}$ clusters. (2) From SAS measurements, the decomposition process at $533 \mathrm{~K}$ was described by nucleation and growth mechanism of the ordered $\delta$ ' phase. At $413 \mathrm{~K}$, the spinodal decomposition of the ordered phase was suggested to take place. (3) The reversion of $\delta$ ' phase is rate-controlled by the dissolution reaction at the interface, while $\delta^{\prime}$ phase was in the ordered state during the process.

\section{Acknowledgements}

The authors wish to thank Prof. Y. Amemiya of KEK for his helpful discussions. They acknowledge the partial financial support of Light Metal Educational Foundation, Inc.

\section{References}

1. L.-Q. Chen and A.G.Khachaturyan, Acta metall. mater., 39(1991), 2533.

2. W.A.Soffa and D.E.Laughlin, Acta metall., 37(1989), 3019.

3. M.-S. Yu and H. Chen, Kinetics of Ordering Transformations in Metals, ed by H.Chen and V.K.Vasudevan (TMS, 1992), p.307.

4. K.Osamura, K.Okuda, M.Tanaka, M.Nagao and Y.Amemiya, ibid, p291.

5. J.S.Garland and J.M.Sanchez, ibid, p.207.

6. H.Okuda and K.Osamura, Acta metall. mater., submitted (1993).

7. H.Okuda, M.Tanaka, K.Osamura and Y.Amemiya, Acta metall. mater., 41(1993), 1733. 